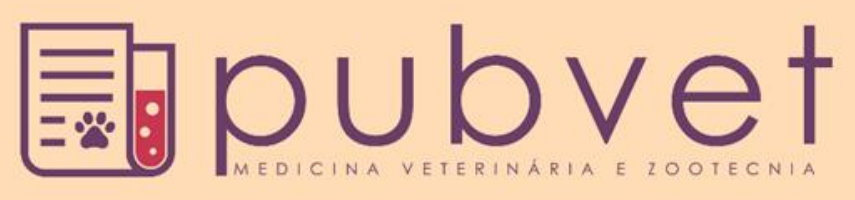

ISSN $1982-1263$

https://doi.org/10.31533/pubvet.v13n6a357.1-6

\title{
Tétano em equino: Relato de caso
}

\begin{abstract}
André Luis de Araújo Pereira ${ }^{1 \oplus,}$, Talia Fabrício Gonçalves ${ }^{2}$, Jackson Brendo Gomes Dantas ${ }^{2}$, Mariana Picoli Martins de Oliveira ${ }^{2} \theta$, Joaquim Bezerra Gomes ${ }^{2} \theta$, Mikael Leandro Duarte de Lima Tolentino $^{3}$, Everton Almeida Pereira $^{3 \oplus}$, Karolynne de Freitas Martins e Silva ${ }^{4}$, Denise Cerqueira de $\operatorname{Sousa}^{50}$, Manoel Lopes da Silva Filho ${ }^{5^{*}}$
\end{abstract}

${ }^{1}$ Medico Veterinário, Autônomo, Bom Jesus, PI, Brasil

${ }^{2}$ Graduando em Medicina Veterinária, Universidade Federal do Piauí, UFPI, Bom Jesus, PI, Brasil

${ }^{3}$ Médico Veterinário do HVU, Universidade Federal do Piauí - UFPI, Bom Jesus, PI, Brasil

${ }^{4}$ Mestra em Zootecnia, Universidade Federal do Piauí, Bom Jesus-PI, Brasil

${ }^{5}$ Professor(a), Departamento de Medicina Veterinária, Universidade Federal do Piauí - UFPI, Bom Jesus, PI, Brasil

*Autor para Correspondência: E-mail:manoellopes@ufpi.edu.br

Resumo. Este trabalho objetivou relatar o caso clínico de uma fêmea equina da raça Paint horse, de aproximadamente seis anos de idade, atendido no Hospital Veterinário Universitário da Universidade Federal do Piauí, com quadro clínico de tétano. Doença causada pelas toxinas, tetanospasmina e tetanolisina, produzidas pela bactéria grampositiva e anaeróbia Clostridium tetani. Por ser uma doença tóxica de alto poder de infecção, ela pode acometer algumas espécies domésticas, porém sua epidemiologia está mais ligada a equinos. Para que ocorra a infecção e surgimento dos sinais clínicos da doença, é necessário que haja uma porta de entrada, como um ferimento, ou contato direto com secreções de outros animais já infectados. O animal uma vez infectado apresenta sinais clínicos como: rigidez dos membros, trismo labial, rigidez muscular, espasmos musculares, hiperestesia e levantar da cauda. Diante dos sinais clínicos apresentados, diagnosticou-se um caso de tétano. Iniciou-se em seguida o tratamento, utilizando-se metronidazol comprimidos na dose de $25 \mathrm{mg} / \mathrm{kg}$, a cada 8 horas durante 5 dias; tiocolchicosídeo comprimidos na dose de $0,05 \mathrm{mg} / \mathrm{kg} / \mathrm{SID}$ durante 5 dias; soro antitetânico $(50.000$ UI/animal) a cada 24 horas por 5 dias; e realizando-se a lavagem da ferida do membro posterior direito diariamente com água, água oxigenada e aplicação tópica de fenitrothion por sete dias consecutivos. A melhora foi gradativa e significativa com o animal respondendo muito bem ao tratamento, obtendo resultados esperados, restabelecendo a saúde do paciente. Tendo alta médica após sete dias de tratamento intensivo.

Palavras Chave: afecção, equídeo, Clostridium tetani

\section{Equine tetanus: Case report}

Abstract. This work aimed to report the clinical case of a female horse of the Paint horse race, approximately six years old, attended at the University Veterinary Hospital of the Federal University of Piauí, with clinical tetanus. Disease caused by toxins, tetanospasmin and tetanolysin, produced by gram-positive and anaerobic bacteria Clostridium tetani. Because it is a toxic disease with high infection power, it can affect some domestic species, but its epidemiology is more related to equines. For infection to occur and clinical signs of the disease occur, there must be a gateway, such as an injury, or direct contact with secretions from other already infected animals. The animal once infected has clinical signs such as limb rigidity, labial trismus, muscle stiffness, muscle spasms, hyperesthesia, and tail lift. In view of the clinical signs presented, a case of tetanus was diagnosed. The treatment was then started using metronidazole tablets at a dose of $25 \mathrm{mg} / \mathrm{kg}$, every 8 hours 
for 5 days; thiocolchicoside tablets at a dose of $0.05 \mathrm{mg} / \mathrm{kg} / \mathrm{SID}$ for 5 days; tetanus serum (50,000 IU/animal), every 24 hours for 5 days; and washing the right posterior limb wound daily with water, hydrogen peroxide and topical application of fenitrothion for seven consecutive days. The improvement was gradual and significant with the animal responding very well to the treatment, obtaining expected results, restoring the patient's health. After being discharged after seven days of intensive treatment.

Keywords: affection, equine, Clostridium tetani

\section{Tétano en equino: Reporte de un caso}

Resumen. Este trabajo objetivó relatar el caso clínico de una hembra equina de la raza Paint horse, de aproximadamente seis años, atendida en el Hospital Veterinario Universitario de la Universidad Federal de Piauí, con cuadro clínico de tétano. Enfermedad causada por las toxinas, tetanaspasmina y tetanolisina, producidas por la bacteria grampositiva y anaeróbica Clostridium tetani. Por ser una enfermedad tóxica de alto poder de infección, puede acometer algunas especies domésticas, pero su epidemiología está más ligada a los equinos. Para que ocurra la infección y surgimiento de los signos clínicos de la enfermedad, es necesario que haya una puerta de entrada, como una herida, o contacto directo con secreciones de otros animales ya infectados. El animal una vez infectado presenta signos clínicos como: rigidez de los miembros, trismo labial, rigidez muscular, espasmos musculares, hiperestesia y levantamiento de la cola. Ante los signos clínicos presentados, se diagnosticó un caso de tétano. Se inició luego el tratamiento, utilizando metronidazol comprimidos a la dosis de $25 \mathrm{mg} / \mathrm{kg}$, cada 8 horas durante 5 días; tiocolchicosídeos comprimidos en la dosis de $0,05 \mathrm{mg} / \mathrm{kg} / \mathrm{SID}$ durante 5 días; suero antitetánico (50.000 UI / animal), cada 24 horas por 5 días; y realizándose el lavado de la herida del miembro posterior derecho diariamente con agua, agua oxigenada y aplicación tópica de fenitrothion por siete días consecutivos. La mejora fue gradual y significativa con el animal respondiendo muy bien al tratamiento, obteniendo resultados esperados, restableciendo la salud del paciente. Teniendo alta médica después de siete días de tratamiento intensivo.

Palabras clave: afección, equino, Clostridium tetani

\section{Introdução}

O tétano é considerado uma toxi-infecção, já que o Clostridium tetani (C. tetani) produz as toxinas que desencadeiam a doença (Green et al., 1994; Thomassian, 2005; Zappa \& Francisco, 2013). É uma enfermidade de grande importância na clínica veterinária, devido à elevada taxa de mortalidade e longo período de convalescença (Smith, 2006). Caracteriza-se por rigidez muscular que pode levar o animal afetado a morte, devido ao seu grau de complicação e severidade dos sintomas apresentados (Smith \& George, 2006), causada pela bactéria gram-positiva e anaeróbia formadora de esporos em sua forma vegetativa, C. tetani (Lima et al., 2013). Os esporos da bactéria são detectados nos solos de lugares de clima temperado e no trato digestivo de animais e humanos, sendo mais frequentes em áreas de solos ricos em fezes de animais (Songer, 1997).

O C. tetani libera no organismo as toxinas tetanospasmina e tetanolisina. A tetanolisina promove a disseminação da infecção, à medida que aumenta a quantidade de necrose tissular local (Smith, 2006; Smith \& George, 2006). E a tetanospasmina é responsável pelo desencadeamento dos sinais do tétano. Assim que a tetanospasmina é liberada pelas células bacterianas, por ação de uma protease, se dividida em duas estruturas, uma cadeia leve e uma cadeia pesada, ligadas por uma ponte dissulfídrica. A toxina, então, atinge o neurônio motor, através da cadeia pesada, é transportada até a medula espinhal e adentra o neurônio inibitório, quando perde a ponte dissulfídrica e se torna ativa. Após a ativação, a cadeia leve age no citoplasma da célula nervosa quebrando as proteínas celulares e impedindo a liberação dos neurotransmissores modulatórios (Guilfoile \& Babcock, 2008). 
A infecção acontece por conta de lesões que causam a morte do tecido vitalizado, como feridas nas regiões mais distais dos membros de equinos submetidos a práticas de manejo inadequado (Radostits et al., 2010; Smith \& George, 2006).

Objetivou-se relatar um caso clínico de tétano equino atendido no Hospital Veterinário Universitário da Universidade Federal do Piauí.

\section{Relato do caso}

Uma égua de aproximadamente seis anos de idade, da raça Paint horse, pesando $300 \mathrm{~kg}$, foi atendida no Hospital Veterinário Universitário da Universidade Federal do Piauí. De acordo com a anamnese o proprietário do animal relatou que a égua supostamente foi picada por uma cobra, perdeu a gestação e apresentava dificuldade para se alimentar. Não era vacinado e nem vermifugado.

Ao exame físico o animal apresentou sinais de hipersensibilidade à palpação e lacerações na pele no terço médio do tendão flexor digital superficial do membro pélvico e no terço proximal do metacarpo do membro torácico direito (Figuras 1A e 1B), frequência cardíaca de 60 batimentos por minuto (bpm), frequência respiratória de 28 movimentos por minuto $(\mathrm{mpm})$, motilidade intestinal diminuída, temperatura de $38,3^{\circ} \mathrm{C}$ e tempo de preenchimento capilar de três segundos e, concomitante a isso, mucosas oculares congestas, protrusão de terceira pálpebra no olho direito, leve rigidez muscular, dificuldade de locomoção, cauda em bandeira, sudorese intensa e orelhas eretas e imóveis.

Diante dos sinais clínicos apresentados pelo equino, diagnosticou-se um caso de tétano. Foi iniciado o protocolo para tratamento, utilizando-se metronidazol comprimidos na dose de $25 \mathrm{mg} / \mathrm{Kg}$, a cada 8 horas durante cinco dias; tiocolchicosídeo comprimidos na dose de $0,05 \mathrm{mg} / \mathrm{Kg} / \mathrm{SID}$ durante 5 dias; soro antitetânico (50.000 UI/animal) a cada 24 horas por 5 dias; e realizando-se a lavagem da ferida diariamente com água, água oxigenada e aplicação tópica de fenitrothion. $\mathrm{O}$ tratamento foi o mesmo todos os dias durante sete dias para que garantisse a eficácia do protocolo estipulado.

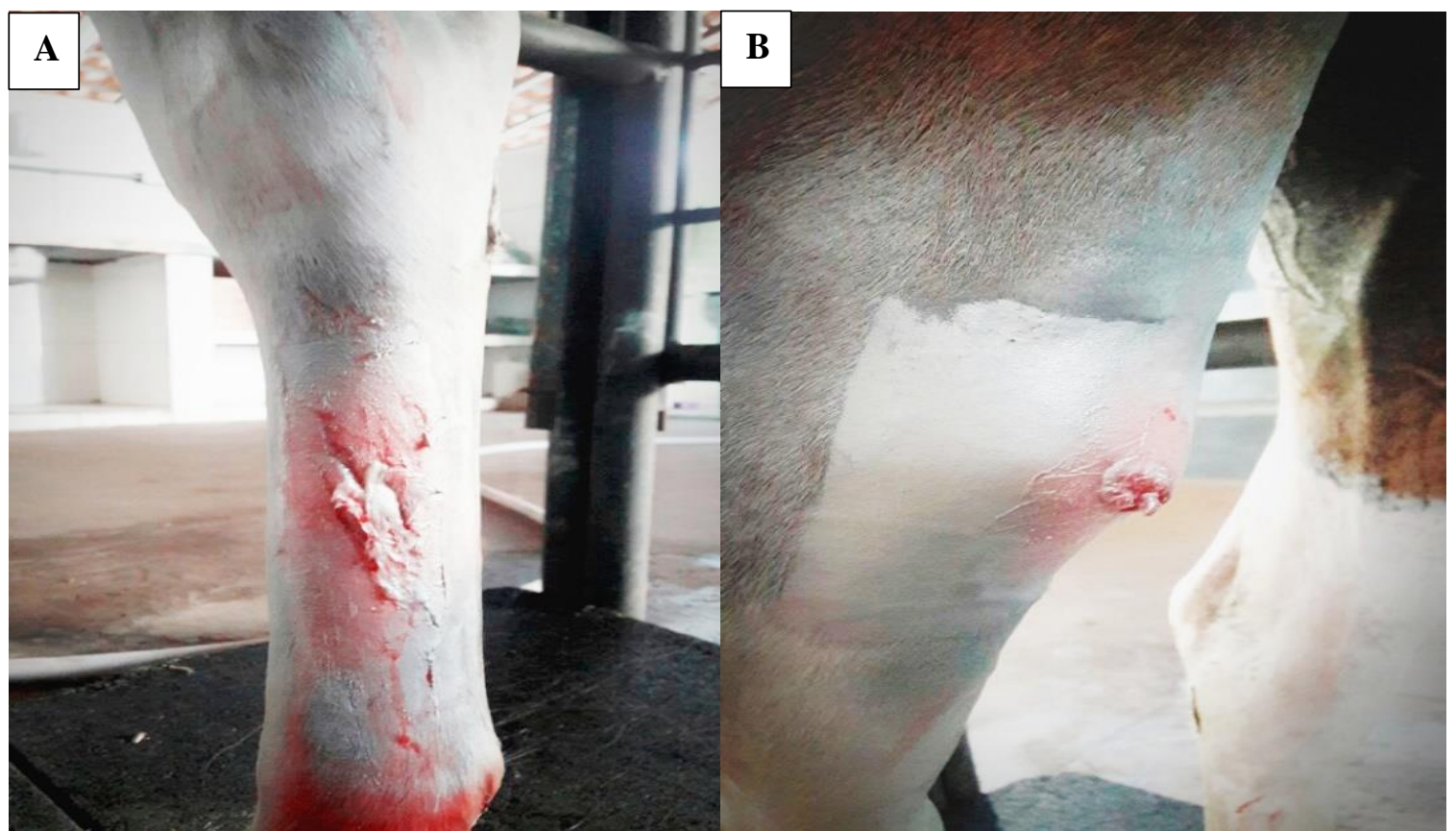

Figura 1. Membro pélvico e Membro torácico direito (figuras A e B), equino, fêmea, da raça Paint horse, seis anos de idade, diagnosticado com tétano, lesões antes do tratamento.

Concomitante ao tratamento o animal foi mantido em uma baia evitando ao máximo estresse físico para garantir um bom resultado do tratamento.

A melhora foi gradativa e significativa com o animal respondendo muito bem ao tratamento, obtendo-se bons resultados, restabelecendo a saúde da paciente (Figuras 2 e 3). Recebendo alta médica após sete dias de tratamento intensivo. 


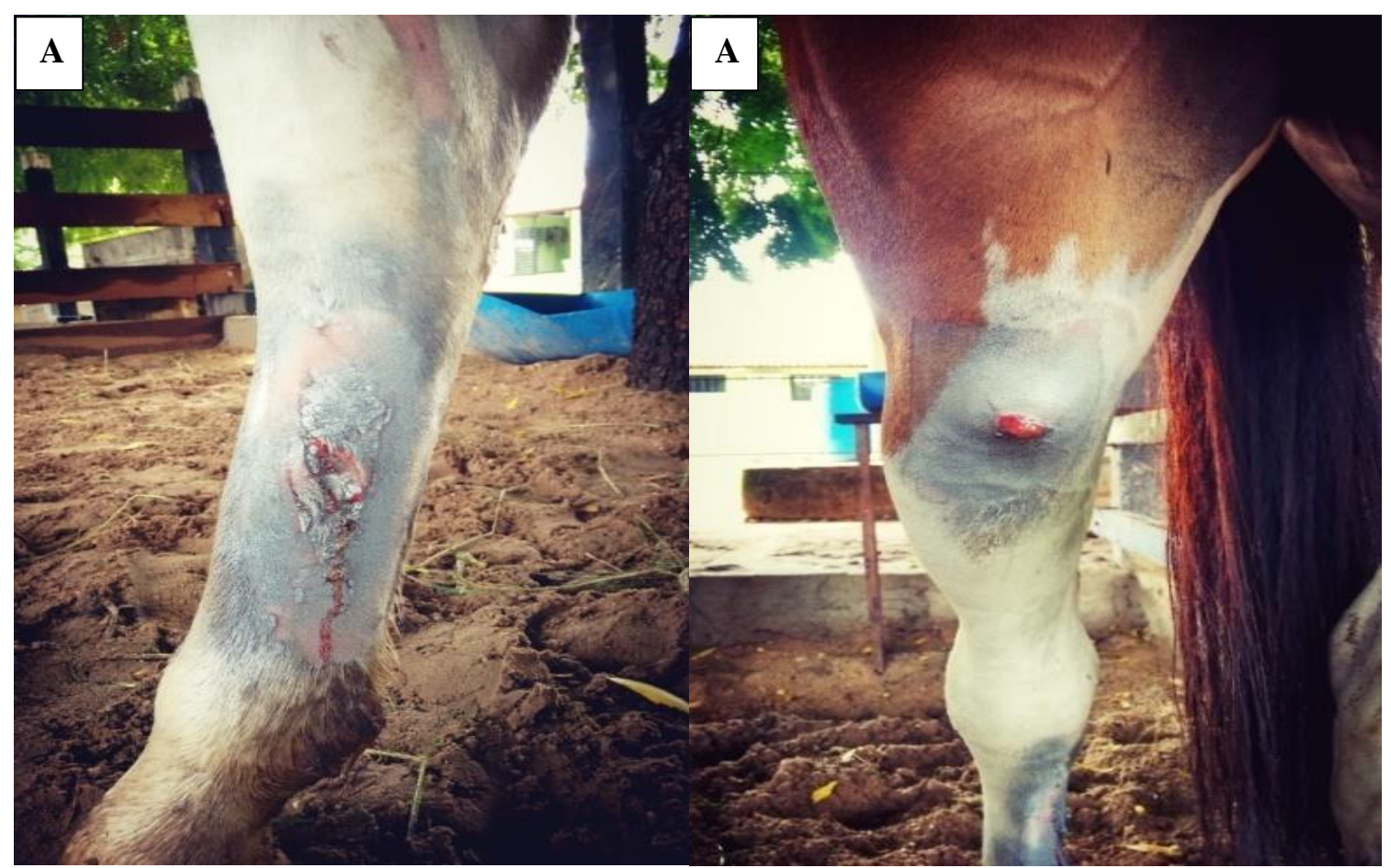

Figura 2. Membro pélvico e membro torácico direito (Figuras A e B), equino, fêmea, da raça Paint horse, seis anos de idade, diagnosticado com tétano, lesões após 7 dias de tratamento.

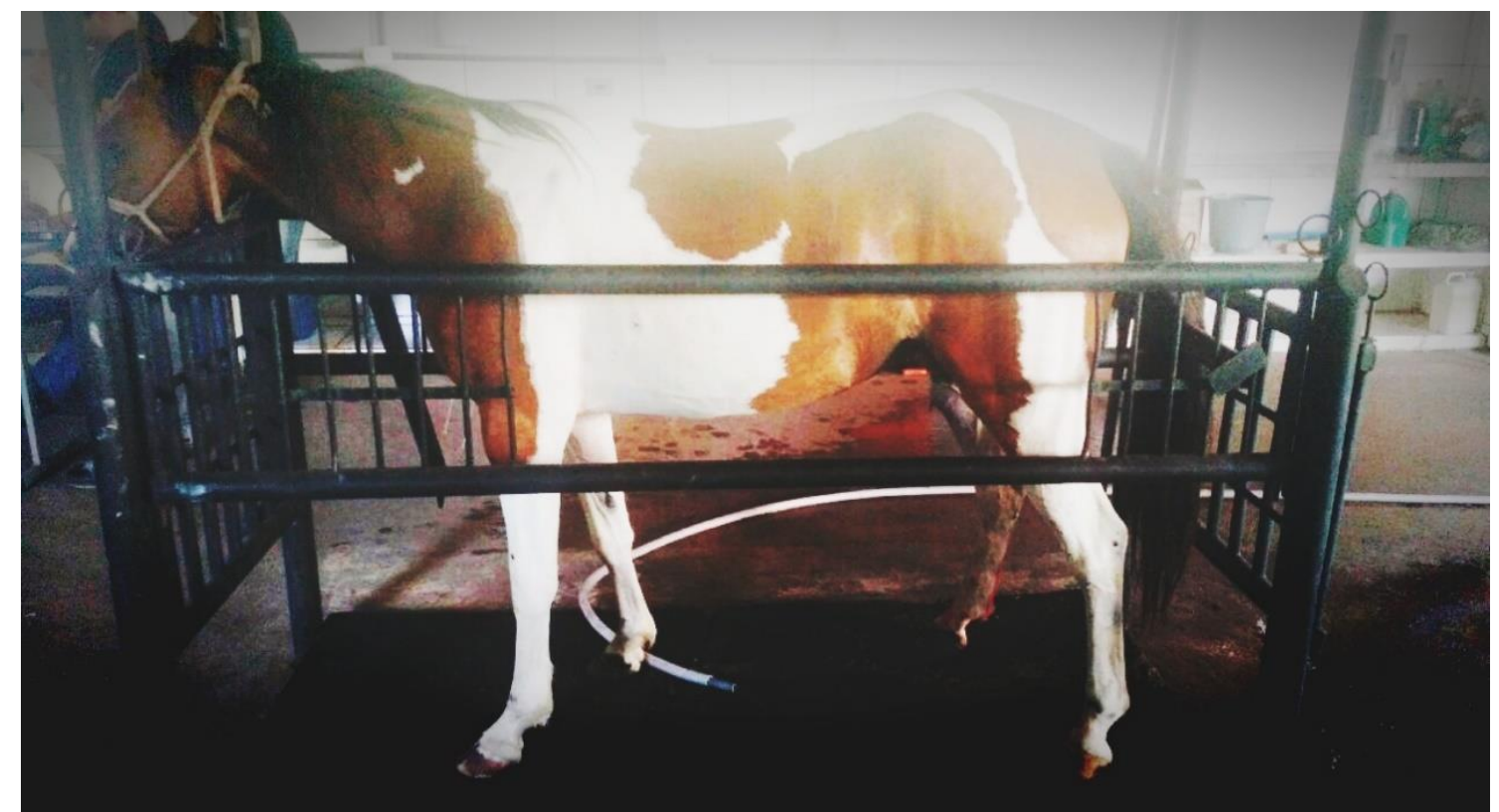

Figura 3. Equino, fêmea, da raça Paint horse, seis anos de idade, após sete dias de tratamento para tétano, demonstrando recuperação do estado clínico apresentado.

\section{Discussão}

O tétano tem como porta de entrada no organismo alguma ferida profunda e penetrante (Radostits et al., 2010). No caso clínico relatado a via de entrada para o C. tetani no organismo foi uma lesão na região do terço médio do tendão flexor digital superficial direito e no terço proximal do metacarpo esquerdo, sendo este um fator de risco para a inoculação e a multiplicação do agente no hospedeiro, assim como descrito por (Lima et al., 2013).

As toxinas produzidas pelo $C$. tetani acabam desencadeando o aparecimento dos sinais clínicos (Thomassian, 2005) que são gradativos e varia de acordo com alguns fatores, como o tempo de infecção, tamanho da lesão, a quantidade de bactérias e até mesmo a capacidade do animal em debelar uma 
infecção espontaneamente pelo seu próprio mecanismo de defesa (Smith \& George, 2006). De uma forma geral, o período de incubação pode variar desde três dias a dois meses, de acordo com Delguste et al. (2008).

Difícil apreensão de alimento, dificuldade para efetuar a micção e rigidez podem ser observados no início da doença, causados por uma rigidez primária que envolve, frequentemente, os músculos massetéricos e do pescoço e os membros pélvicos (Delguste et al., 2008), como observado no relato de caso em questão. Os reflexos ficam mais evidenciados após o primeiro dia de infecção por C. tetani.

Geralmente o prognóstico para a doença é reservado. Iniciar rapidamente o tratamento após a identificação dos primeiros sinais e tempo de hospitalização igual ou maior que sete dias, são cruciais para um melhor prognóstico. Do contrário, disfagia, dispneia, decúbito e lesões perfurantes na sola do casco podem gerar um prognóstico desfavorável (Delguste et al., 2008; Pimentel et al., 2009; Reichmann et al., 2008).

O controle e eliminação da bactéria, C. tetani, acontece por meio de antibioticoterapia, e geralmente se usa penicilina G via parenteral (Radostits et al., 2010). Todavia, de acordo MacKay (2005), o uso de penicilina no tratamento de tétano não deve ser utilizado, devido ser um antagonista do GABA (ácido gama-aminobutírico), utilizando assim por sua vez o metronidazol, na dose de $25 \mathrm{mg} / \mathrm{kg}$, BID, IV, VO. Corroborando com este estudo onde se utilizou o metronidazol associado com o tiocolchicosídeo.

No local deve ser feito uma limpeza de toda a lesão realizando debridamento e uso de água oxigenada para desinfecção. O uso tópico de penicilina no local da ferida pode ser realizado (Radostits et al., 2010). Para auxílio no tratamento do tétano é fundamental o uso de soro antitetânico. Recomenda-se a administração única de 5000 UI/animal subcutânea, até 2.500.000 UI/animal, posteriormente aplicação de doses inferiores durante cinco dias (Green et al., 1994). Sendo este procedimento utilizado neste tratamento.

Para controlar os espasmos musculares e rigidez dos mesmos fazem-se o uso de outros fármacos com ação sedativa e/ou relaxantes musculares. A droga de eleição para relaxamento muscular em caso de tétano é a Acepromazina na dose de 0,05 a $0,1 \mathrm{mg} / \mathrm{kg}$, a cada 6 horas até que seja perceptível a diminuição da rigidez muscular e cause leve sedação (Mayhew, 2009). O Diazepam na dose de 0,05-0,4 $\mathrm{mg} / \mathrm{kg}$ e o Metocarbamol 10-20 mg/kg também ajudam no relaxamento muscular (Beasley, 1999).

No caso clínico descrito, o diagnóstico foi baseado no histórico e sinais clínicos corroborando com Radostits et al. (2010) que caracteriza o tétano como uma doença de fácil diagnóstico com base no histórico e a presença dos sinais clínicos patognomônicos como, por exemplo, o prolapso de terceira pálpebra. Nos últimos anos, a casuística de casos clínicos de tétano em equinos, tem diminuído devido à utilização da prevenção e profilaxia com vacinas que conferem imunização após 3 a 4 semanas necessitando de reforço anual (Heldens et al., 2010). Diferindo do caso acompanhado onde o animal não era vacinado.

\section{Conclusão}

O tétano em equinos, quando tratado logo nos primeiros sinais de manifestação clínica da doença, apresentam resultados satisfatórios.

\section{Referências bibliográficas}

Beasley, V. R. (1999). Toxicants associated with CNS stimulation or seizures. A Systems Affected Approach to Veterinary Toxicology94-97.

Delguste, C., Sandersen, C., Verwilghen, D., Grulke, S. \& Amory, H. (2008). Tetanus in the equine species: a retrospective study of 31 cases. Tijdschrift Voor Diergeneeskunde, 133(12):512-517.

Green, S. L., Little, C. B., Bairad, J. D., Tremblay, R. R. M. \& Smithmaxie, L. L. (1994). Tetanus in the horse: A review of 20 casos (1970-1990). Journal Veterinary Internal Medicine, 8128-132.

Guilfoile, P. \& Babcock, H. (2008). Tetanus. New York, US: Chelsea House. 
Heldens, J. G. M., Pouwels, H. G. W., Derks, C. G. G., Van de Zande, S. M. A. \& Hoeijmakers, M. J. H. (2010). Duration of immunity induced by an equine influenza and tetanus combination vaccine formulation adjuvanted with ISCOM-Matrix. Vaccine, 28(43):6989-6996.

Lima, J. T. B., Patrício, L. A. M. M., Amorim , F. A. F., Santos, S. G. \& Baptista Filho, L. C. F. B. (2013). Tétano em equino - relato de caso. Paper presented at the Jornada de ensino, pesquisa e extensão, Recife.

MacKay, R. J. (2005). Neurologic disorders of neonatal foals. Veterinary Clinics: Equine Practice, 21(2):387-406.

Mayhew, J. (2009). Tetany, tremor and postural and movement disorders. Paper presented at the 1th International Congress of World Equine Veterinary Association, Guarujá, São Paulo, Brasil.

Pimentel, L. A., Oliveira, D. M., Galiza, G. J. N., Rego, R. O., Dantas, A. F. M. \& Riet-Correa, F. (2009). Doenças do sistema nervoso central de equídeos no semi-árido. Pesq. Vet. Bras, 29(7):589597.

Radostits, O. M., Gay, C. C., Blood, D. C., Hinchcliff, K. W. \& McKenzie, R. A. (2010). Clínica Veterinária: um tratado de doenças dos bovinos, ovinos, suínos, caprinos e eqüinos (Vol. 1). Rio de Janeiro: Guanabara Koogan.

Reichmann, P., Lisboa, J. A. N. \& Araujo, R. G. (2008). Tetanus in equids: A review of 76 cases. Journal of Equine Veterinary Science, 28(9):518-523.

Smith, M. O. (2006). Tratado de medicina interna de grandes animais (Vol. 1). São Paulo: Manole.

Smith, M. O. \& George, L. W. (2006). Enfermidades do sistema nervoso. In B. P. Smith (Ed.), Medicina interna de grandes animais (pp. 972-1111). Barueri, São Paulo, Brasil: Manole.

Songer, J. G. (1997). Clostridial diseases in animals. In J. I. Rood, B. A. McClane, J. G. Songer \& R. W. Titball (Eds.), The Clostridia: molecular biology and pathogenesis (pp. 153-182). San Diego, USA: Academic Press.

Thomassian, A. (2005). Enfermidades dos cavalos. Sao Paulo, Brasil: Livraria Varela.

Zappa, V. \& Francisco, L. (2013). Tétano em equinos - Revisão de literatura. Revista Científica Eletrônica de Medicina Veterinária, 21(1):1-7.

Recebido: 6 de maio, 2019.

Aprovado: 30 de maio, 2019.

Publicado: 30 de junho, 2019.

Licenciamento: Este artigo é publicado na modalidade Acesso Aberto sob a licença Creative Commons Atribuição 4.0 (CCBY 4.0), a qual permite uso irrestrito, distribuição, reprodução em qualquer meio, desde que o autor e a fonte sejam devidamente creditados. 